\title{
Diabetes and mortality in patients with COVID-19: Are we missing the link?
}

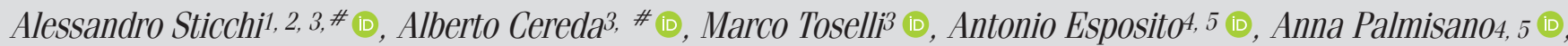 \\ Davide Vignalet, 5 (D), Valeria Nicolettit, 5 (D), Riccardo Leonet, 5 (D), Chiara Gnassot, 5 (D), Alberto Monello 6 (D), \\ Arif A. Khokhar ${ }^{3}$ (D), Alessandra Laricchia ${ }^{3}$ (D), Andrea Biagi6 (D), Piergiorgio Turchio 6 (D), Marcello Petrini 6 (D),

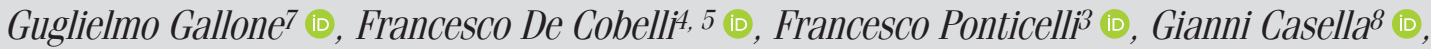 \\ Gianmarco Iannopollo 8 (D), Tommaso Nanninis (D), Carlo Tacchettit, 5 (D), Antonio Colombo ${ }^{3}$ (D), Francesco Gianninis (i) \\ ${ }^{1}$ Centro per la Lotta Contro L'Infarto (CLI) Foundation; Rome-Italy \\ ${ }^{2}$ Saint Camillus International University of Health Sciences; Rome-Italy \\ ${ }^{3} \mathrm{GVM}$ Care \& Research Maria Cecilia Hospital; Cotignola-Italy \\ ${ }^{4}$ School of Medicine, Vita-Salute San Raffaele University; Milan-Italy \\ ${ }^{5}$ Experimental Imaging Center, IRCCS San Raffaele Scientific Institute; Milan-Italy \\ ${ }^{6}$ Guglielmo da Saliceto Hospital; Piacenza-Italy \\ ${ }^{7}$ Division of Cardiology, Department of Internal Medicine, Città della Salute e della Scienza; Turin-Italy \\ ${ }^{8}$ Maggiore Hospital; Bologna-Italy
}

Cite this article as: Sticchi A, Cereda A, Toselli M, Esposito A, Palmisano A, Vignale D, et al. Diabetes and mortality in patients with COVID-19: Are we missing the link? Anatol J Cardiol 2021; 25: 376-9.

\section{Introduction}

The coronavirus disease 2019 (COVID-19) outbreak was the first pandemic of the $21^{\text {st }}$ century, and healthcare systems around the world are struggling to cope with this emergency (1). Understanding which patients are at higher risk of severe infection and complications is a priority, both to optimize resource allocation and to develop specific tailored management strategies.

Patients affected by diabetes seem to be at increased risk of poor outcomes following COVID-19 infection (1-3). In a large observational study in China, the mortality rate of patients with diabetes was three times higher than that of nondiabetics. A recent meta-analysis showed a two-fold risk in terms of mortality, severity, and progression to acute respiratory distress syndrome in patients with diabetes affected by COVID-19 $(2,3)$.

Many potential mechanisms of adverse outcomes in patients with diabetes with COVID-19 infection have been hypothesized (4). However, the pathobiological link between diabetes and the severity of the COVID-19 disease course remains unclear $(4,5)$. Although the association of diabetes with COVID-19 mortality has been proven independently from several disease predictors, including the burden of cardiovascular risk factors, whether it is also independent from the actual presence and extent of atherosclerosis remains unsettled.

Non-cardiosynchronized chest computed tomography (CT), frequently essential among patients with COVID-19, allows the acquisition of multiple abnormalities, including interstitial lung involvement, pleuro-pericardial effusion, and cardiovascular calcifications.

\section{Research design}

We assessed the interaction between cardiovascular atherosclerotic disease burden and type II diabetes in 1,656 consecutive patients hospitalized for COVID-19 who underwent chest CT for assessment of pneumonia severity from the Italian multicenter registry SCORE-COVID (Calcium Score for COVID-19 Risk Evaluation). The type I diabetes population was excluded because it represented a small number of patients and a potential confounding factor in the analysis. CT scans were acquired during the most critical phase of the pandemic in Italy (between

\footnotetext{
\#The two authors contributed equally.

Address for Correspondence: Alessandro Sticchi, MD, Centro per la Lotta Contro L'Infarto (CLI) Foundation, Saint Camillus International University of Health Sciences, Rome; GVM Care \& Research, Maria Cecilia Hospital, Cotignola-Italy Phone: +393270859187 E-mail: sticchialessandro@gmail.com Accepted Date: 08.04.2021 Available Online Date: 21.05.2021 


\section{HIGHLIGHTS}

- In patients with coronavirus disease 2019 (COVID-19), diabetes seems to represent a significant risk factor in determining poor outcomes. However, the mechanisms of this interaction need further investigations to be clarified.

- In a large registry of all-comers patients with COVID-19 (Italian SCORE-COVID registry), cardiovascular risk burden, derived by computed tomography findings, and demographic cardiovascular risk factors appeared to have a superior prognostic role than diabetes.

- Patients with COVID-19 at high risk for cardiovascular burden should be carefully evaluated similarly to patients with diabetes, considering the potential poor events demonstrated in our study cohort. Further studies need to clarify the role of diabetes type II in COVID-19 infection.

March 2020 and April 2020) across 16 second- and third-level hospitals (Table 1).

\section{Methods}

The burden of cardiovascular atherosclerotic disease was defined by the volume of calcifications involving the thoracic cardiovascular system, including coronary arteries, aortic valve, and thoracic aorta. It was quantified on the CT exam by an operator blinded to clinical data. The portion of lung volume involved in the inflammatory process was also quantified. The primary endpoint was in-hospital mortality. Unpaired $t$-test was used for comparisons of continuous variables, and chi-square test was used for categorical variables. Kaplan-Meier estimates were performed to evaluate cumulative in-hospital mortality rates at follow-up. The interaction of atherosclerotic disease burden with diabetes was then tested with a multivariate Cox proportional hazards model, also including age, sex, creatinine, and pneumonia lung involvement.

\section{Results}

Baseline characteristics stratified by diabetes status are presented in Table 1. The prevalence of diabetes was 19.3\% among hospitalized patients with COVID-19, consistent with previous reports (1). Patients with diabetes were older (1) $(71 \pm 11$ vs. $66 \pm 14$ years, $p=0.001$ ) and had a higher burden of cardiovascular risk factors and atherosclerotic disease [coronary artery calcium (CAC) volume: $643 \pm 1,158 \mathrm{~mm}^{3}$ vs. $257 \pm 634 \mathrm{~mm}^{3}, \mathrm{p}=0.001$ ] Greater lung involvement at the chest CT was also observed (aerated lung volume: $2,244 \pm 1,286 \mathrm{~mm}^{3}$ vs. $2,465 \pm 1,444 \mathrm{~mm}^{3}$, $\mathrm{p}=0.012$ ).

Patients with diabetes experienced a higher incidence of stroke $(5.6 \%$ vs. $2.1 \%, p=0.010)$ and acute coronary syndromes $(3.4 \%$ vs. $1.1 \%, p=0.022)$ but no difference in peripheral arterial

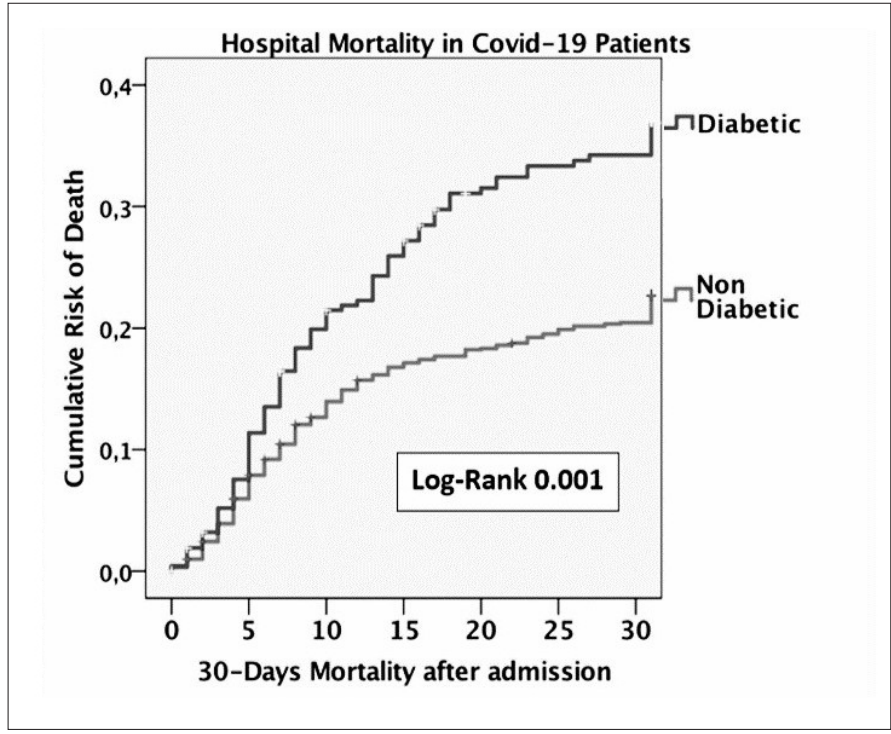

Figure 1. Kaplan-Meier survival curve for type 2 diabetes

embolization and pulmonary embolism incidence $(6.2 \%$ vs. $5.6 \%$, $\mathrm{p}=0.730$ ). Diabetics were also more frequently admitted to intensive care unit (ICU) $(57.7 \%$ vs. $45.8 \%, p=0.001)$, although with similar intubation occurrence ( 11.9 vs. $12.3 \%, p=0.480)$ compared with nondiabetic patients.

In-hospital mortality was significantly higher in diabetics [30.4 vs. $20.1 \%, p=0.001$; unadjusted hazard ratio (HR): $1.61,95 \%$ confidence interval (CI): 1.28-2.04, log-rank=0.001; Fig. 1]. In multivariate analysis (Table 2), diabetes was not associated with in-hospital mortality (HR: 1.141, 95\% Cl: $0.869-1.498, p=0.343$ ), whereas the calcification volume was a significant outcome predictor (HR: 1, 95\% Cl: 1.000-1.001, $\mathrm{p}=0.034$ ).

\section{Conclusions}

Our findings downsize the meaning of the prognostic role of diabetes in patients with COVID-19. We investigated the role of diabetes independently from the cardiovascular risk profile (expressed by age, sex, creatinine, and CAC) and the extension of pneumonia, demonstrating a superior predictive role of the other variables compared with diabetes alone.

Several studies and meta-analysis correlated diabetes with the severity of COVID-19 disease $(5,6)$, as also shown by our results, both in terms of overall mortality and ICU admission. Although diabetes remains an important factor to risk stratify patients with COVID-19 and to tailor specific management (especially when steroid therapy is needed), several implications may be derived from our findings. First, our data suggest a role for diabetes as a promoter of cardiovascular vulnerability, rather than as an independent prognostic driver. This is of particular relevance for the development of tailored treatment strategies in COVID-19, which should rather be directed at mitigating the negative impact of atherosclerotic disease rather than at diabetes. This hypothesis is supported by the lower severity of COVID-19 progress in patients with type 1 diabetes 


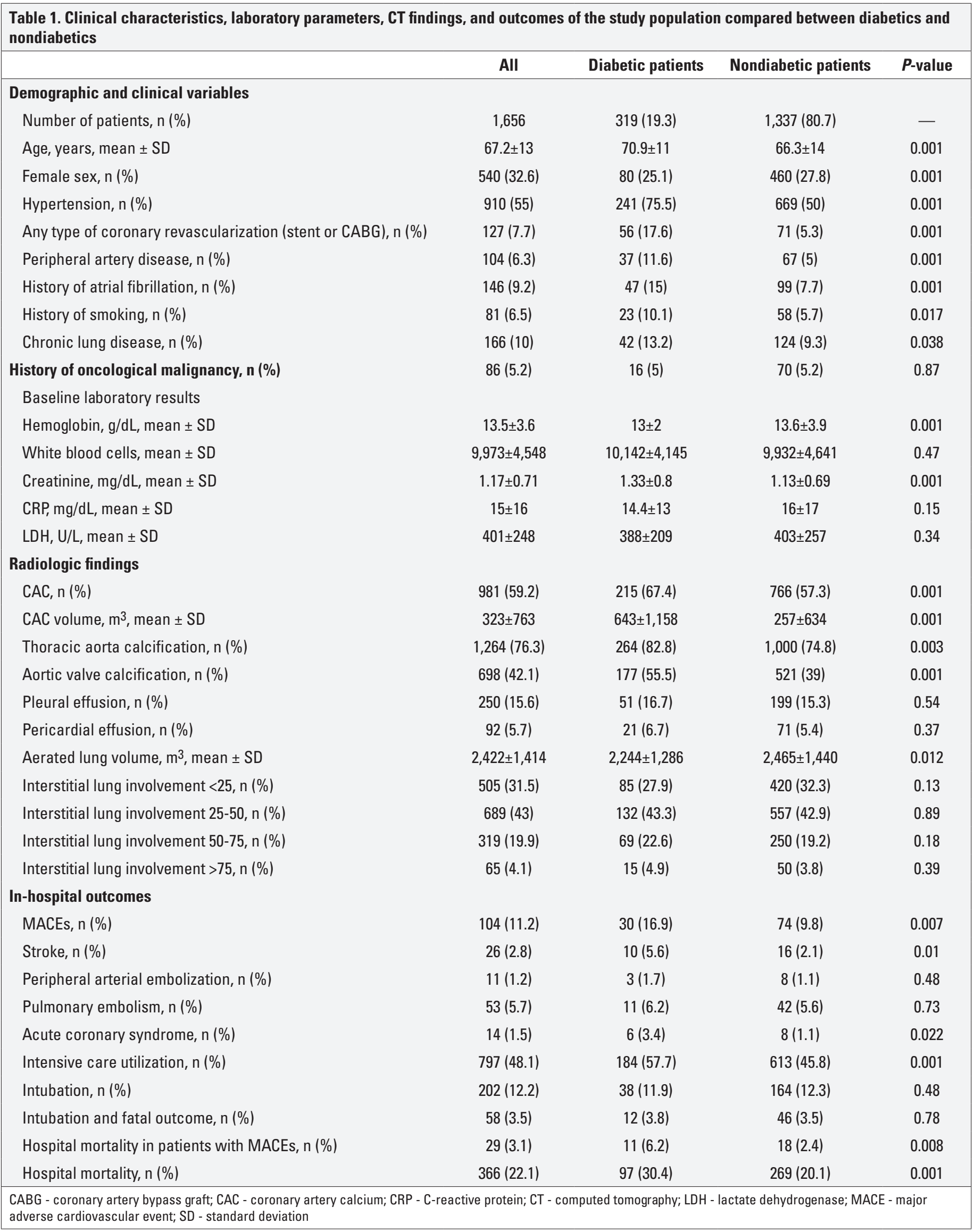




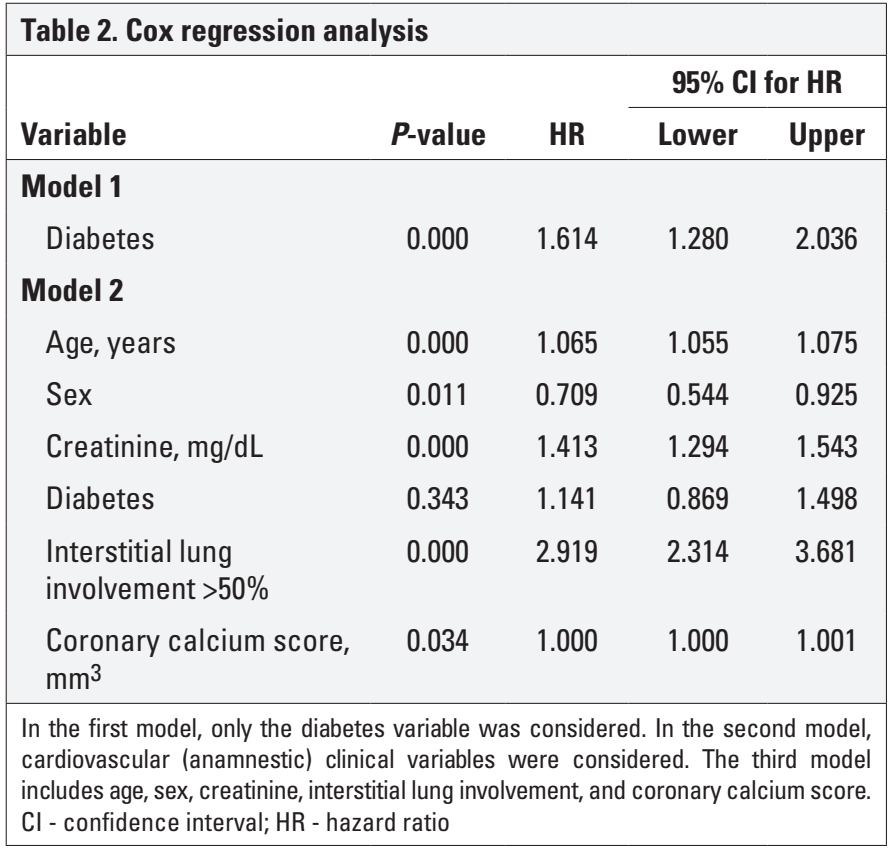

because of an imbalance between the two types of immunity and cardiovascular damage gathered $(1,2,4,7)$. In contrast, some authors show a weak significance of this worse outcome [odds ratio (OR): $3.12 ; 95 \% \mathrm{Cl}: 1.0-9.75 ; \mathrm{p}=0.05$ ] and for ICU admission (OR: 2.72; 95\% Cl: 0.70-10.6; $\mathrm{p}=0.15)(8,9)$. Second, although limited by the observational study design, our results compellingly suggest the potential additive role of CT-derived thoracic cardiovascular calcifications in the risk stratification of patients with COVID-19 over the clinical risk profile.

Future mechanistic studies are warranted to validate our findings by clarifying the pathobiological link among diabetes, atherosclerosis, and COVID-19 and providing a rationale for tailored management strategies.

Conflict of interest: None declared.

Peer-review: Externally peer-reviewed.

Author contributions: Concept - A.S., A.Cereda, M.T., A.E., A.P., D.V., V.N., R.L., C.G., A. M., A.A.K., A.L., A.B., P.T., M.P., G.G., F.D.C., F.P., G.C., G. I., T.N., C.T., A.Colombo, F.G.; Design - A.S., A.Cereda, M.T., A.E., A.P., D.V., V.N., R.L., C.G., A. M., A.A.K., A.L., A.B., P.T., M.P., G.G., F.D.C., F.P., G.C., G.
I., T.N., C.T., A.Colombo, F.G.; Supervision - A.S., A.Cereda, M.T., A.E., A.P., D.V., V.N., R.L., C.G., A. M., A.A.K., A.L., A.B., P.T., M.P., G.G., F.D.C., F.P., G.C., G. I., T.N., C.T., A.Colombo, F.G.; Fundings - None; Materials - None; Data collection \&/or processing - A.E., A.P., D.V., V.N., R.L., C.G., A. M., A.A.K., A.L., A.B., P.T., M.P., G.G., F.D.C., F.P., G.C., G. I., T.N., C.T.; Analysis \&/or interpretation - A.S., A.Cereda, M.T., A.E., A.P., D.V., V.N., R.L., C.G., A. M., A.A.K., A.L., A.B., P.T., M.P., G.G., F.D.C., F.P., G.C., G. I., T.N., C.T.; Literature search - A.S., A.Cereda; Writing - A.S., A.Cereda, F.G.; Critical review - A.S., A.Cereda, M.T., A.Colombo, F.G.

\section{References}

1. Driggin E, Madhavan MV, Bikdeli B, Chuich T, Laracy J, BiondiZoccai G, et al. Cardiovascular Considerations for Patients, Health Care Workers, and Health Systems During the COVID-19 Pandemic. J Am Coll Cardiol 2020; 75: 2352-71. [Crossref]

2. Huang I, Lim MA, Pranata R. Diabetes mellitus is associated with increased mortality and severity of disease in COVID-19 pneumonia - A systematic review, meta-analysis, and meta-regression. Diabetes Metab Syndr 2020; 14: 395-403. [Crossref]

3. The Novel Coronavirus Pneumonia Emergency Response Epidemiology Team. The Epidemiological Characteristics of an Outbreak of 2019 Novel Coronavirus Diseases (COVID-19) - China, 2020. China CDC Weekly 2020; 2: 113-22. [Crossref]

4. Rubino F, Amiel SA, Zimmet P, Alberti G, Bornstein S, Eckel RH, et al. New-Onset Diabetes in Covid-19. N Engl J Med 2020; 383: 789-90. [Crossref]

5. Zheng Z, Peng F, Xu B, Zhao J, Liu H, Peng J, et al. Risk factors of critical \& mortal COVID-19 cases: A systematic literature review and meta-analysis. J Infect 2020; 81: e16-25. [Crossref]

6. Tian W, Jiang W, Yao J, Nicholson CJ, Li RH, Sigurslid HH, et al. Predictors of mortality in hospitalized COVID-19 patients: A systematic review and meta-analysis. J Med Virol 2020; 92: 1875-83. [Crossref]

7. Tatti $P$, Tonolo $G$, Zanfardino A, lafusco D. Letter to the Editor: CoVid-19 and type 1 diabetes: Every cloud has a silver lining. Searching the reason of a lower aggressiveness of the CoronaVirus disease in type 1 diabetes. Diabetes Res Clin Pract 2020: 108270. [Crossref]

8. Yang J, Zheng Y, Gou X, Pu K, Chen Z, Guo Q, et al. Prevalence of comorbidities and its effects in patients infected with SARS-CoV-2: a systematic review and meta-analysis. Int J Infect Dis 2020; 94: 91-5. [Crossref]

9. Jain V, Yuan JM. Predictive symptoms and comorbidities for severe COVID-19 and intensive care unit admission: a systematic review and meta-analysis. Int J Public Health 2020; 65: 533-46. [Crossref] 